\title{
On Extending the Quasilinearization Method to Higher Order Convergent Hybrid Schemes Using the Spectral Homotopy Analysis Method
}

\author{
Sandile S. Motsa and Precious Sibanda \\ School of Mathematics, Statistics \& Computer Science, University of KwaZulu-Natal, Private Bag X01, Scottsville, \\ Pietermaritzburg 3209, South Africa \\ Correspondence should be addressed to Precious Sibanda; sibandap@ukzn.ac.za
}

Received 22 January 2013; Revised 22 March 2013; Accepted 5 April 2013

Academic Editor: Saeid Abbasbandy

Copyright (C) 2013 S. S. Motsa and P. Sibanda. This is an open access article distributed under the Creative Commons Attribution License, which permits unrestricted use, distribution, and reproduction in any medium, provided the original work is properly cited.

We propose a sequence of highly accurate higher order convergent iterative schemes by embedding the quasilinearization algorithm within a spectral collocation method. The iterative schemes are simple to use and significantly reduce the time and number of iterations required to find solutions of highly nonlinear boundary value problems to any arbitrary level of accuracy. The accuracy and convergence properties of the proposed algorithms are tested numerically by solving three Falkner-Skan type boundary layer flow problems and comparing the results to the most accurate results currently available in the literature. We show, for instance, that precision of up to 29 significant figures can be attained with no more than 5 iterations of each algorithm.

\section{Introduction}

The quasilinearization method (QLM) was originally developed by Bellman and Kalaba [1] as a generalization of the Newton-Raphson method to provide lower and upper bound solutions of nonlinear differential equations. The attraction of quasilinearization is that the algorithm is easy to understand and the method generally converges rapidly if the initial guess is close to the true solution.

Bellman and Kalaba [1] established that the method converges quadratically. However, the original proof of quadratic convergence was subject to restrictive conditions of small step size and convexity or concavity of nonlinear functions, Maleknejad and Najafi [2]. These conditions were subsequently relaxed and the method generalized to be applicable to a wider class of problems; see, for instance, papers by Mandelzweig and his coworkers [3-6] and Lakshmikantham $[7,8]$. Parand et al. [9] used the quasilinearization method to solve Volterra's model for population growth in a closed system. Other uses of the quasilinearization method include application to reaction diffusion equations, Jiang and Vatsala
[10], and to Volterra integro-differential equations, Ahmad [11], Pandit [12], and Ramos [13].

An often noted disadvantage of quasilinearization is the instability of the method whenever a poor initial guess is chosen, Tuffuor and Labadie [14]. To improve the accuracy and convergence of the quasilinearization method for all initial guesses, we propose in this paper to embed the QLM algorithm within the spectral homotopy analysis method (SHAM) to obtain a sequence of integration schemes with arbitrary higher order convergence.

The spectral homotopy analysis method was introduced by Motsa et al. $[15,16]$ to address some limitations of the standard homotopy analysis method of Liao $[17,18]$ by, for example, improving the rate of convergence and extending the region of validity of solutions. The SHAM has been used to solve nonlinear equations that arise in the study of fluid flow problems and other areas of science and engineering, Sibanda et al. [19] and Motsa and Sibanda [20].

Abbasbandy [21] and Chun [22] proposed and studied several methods for nonlinear equations with higher order convergence by using the Adomian decomposition technique 
[23-25]. Higher order Newton-like iteration formulae for the computation of the solutions of nonlinear equations were also derived in Chun [26] using the homotopy analysis method and by [27] using the homotopy perturbation method. In this paper we extend the ideas used for the solution of nonlinear equations to obtain higher order iteration schemes for solving nonlinear boundary value problems. We propose an extension of the quasilinearization method by using the spectral homotopy analysis method within the QLM algorithm to obtain a sequence of highly accurate and convergent higher order iterative schemes for solving boundary value problems. For illustration purposes we have presented three QLMSHAM hybrid iteration schemes that are used to solve the Blasius and Falkner-Skan equations. The results are compared to the most accurate skin friction coefficients currently available in the literature by Boyd [28] and Ganapol [29]. Ganapol [29] used an algorithm based on a Maclaurin series with Wynnepsilon convergence acceleration and analytical continuation to obtain highly accurate skin friction coefficients for the Blasius and Falkner-Skan boundary layer flows. The schemes derived in this paper however require neither convergence acceleration nor analytical continuation to remain steady and accurate for up to 29 digits of precision. In addition, the present schemes are highly efficient with 29-digit precision achieved with five or fewer iterations as compared with at least 104 iterations of Ganapol's algorithm.

The structure of this paper is as follows. Section 2 gives a general framework for the derivation of the hybrid quasilinearization-SHAM schemes for the solution of nonlinear differential equations. Section 3 illustrates the application of the three schemes derived in this paper to the solution of Blasius and Falkner-Skan equations. In Section 4, the results are presented and comparison made with the most accurate skin friction results for Blasius and Falkner-Skan equations currently available in the literature.

\section{Derivation of the Iterative Schemes}

In this section we present a framework for the derivation of general QLM-SHAM iterative schemes for solving onedimensional nonlinear differential equations. We consider a general $n$-order nonlinear ordinary differential equation of the form

$$
\begin{aligned}
& L\left[y(x), y^{(1)}(x), y^{(2)}(x), \ldots, y^{(n)}\right] \\
& \quad+F\left[y(x), y^{(1)}(x), y^{(2)}(x), \ldots, y^{(n)}\right]=\psi(x),
\end{aligned}
$$

where $\psi(x)$ is a known function of the independent variable $x$ and $y(x)$ is an unknown function. The functions $L$ and $F$ represent the linear and nonlinear components of the governing equation, respectively. We assume that (1) is to be solved for $x \in[a, b]$ subject to the boundary conditions

$$
\begin{aligned}
& B_{a}\left(y(a), y^{(1)}(a), \ldots, y^{(n-1)}(a)\right)=0, \\
& B_{b}\left(y(b), y^{(1)}(b), \ldots, y^{(n-1)}(b)\right)=0,
\end{aligned}
$$

where $B_{a}$ and $B_{b}$ are linear operators.
Following $[22,27]$, we assume that the true solution of (1) is $y_{\alpha}(x)$ and that $y_{\gamma}(x)$ is an initial approximation that is sufficiently close to $y_{\alpha}(x)$. After expanding $F$ using Taylor series up to first order about $y_{\gamma}, y_{\gamma}^{\prime}, \ldots, y_{\gamma}^{(n)}$ we obtain the following coupled system:

$$
\begin{aligned}
& L\left[y, y^{(1)}, \ldots, y^{(n)}\right]+F\left[y_{\gamma}, y_{\gamma}^{(1)}, \ldots, y_{\gamma}^{(n)}\right] \\
& \quad+\sum_{s=0}^{n}\left(y^{(s)}-y_{\gamma}^{(s)}\right) \frac{\partial F}{\partial y^{(s)}}(\cdots) \\
& \quad+G\left(y, \ldots, y^{(n)}\right)=\psi(x) \\
& G\left(y, y^{(1)}, \ldots, y^{(n)}\right) \\
& \quad=F\left(y, y^{(1)}, \ldots, y^{(n)}\right)-F\left[y_{\gamma}, y_{\gamma}^{(1)}, \ldots, y_{\gamma}^{(n)}\right] \\
& \quad-\sum_{s=0}^{n}\left(y^{(s)}-y_{\gamma}^{(s)}\right) \frac{\partial F}{\partial y^{(s)}}(\cdots),
\end{aligned}
$$

where, for compactness, $(\cdots)$ denotes $\left(y_{\gamma}, y_{\gamma}^{(1)}, \ldots, y_{\gamma}^{(n)}\right)$. Note that adding (3) and (4) gives (1). Equation (3) can be rewritten in the form

$$
\begin{gathered}
\mathscr{L}_{1}\left[y, y^{(1)}, \ldots, y^{(n)}\right]+\mathscr{G}_{1}\left[y, y^{(1)}, \ldots, y^{(n)}\right] \\
=\Phi\left(y_{\gamma}, y_{\gamma}^{(1)}, \ldots, y_{\gamma}^{(n)}\right),
\end{gathered}
$$

where

$$
\begin{aligned}
\mathscr{L}_{1} & {\left[y, y^{(1)}, \ldots, y^{(n)}\right] } \\
& =L\left[y, y^{(1)}, \ldots, y^{(n)}\right]+\sum_{s=0}^{n} y^{(s)} \frac{\partial F}{\partial y^{(s)}}\left(y_{\gamma}, y_{\gamma}^{(1)}, \ldots, y_{\gamma}^{(n)}\right),
\end{aligned}
$$

$$
\begin{aligned}
\Phi\left(y_{\gamma}, y_{\gamma}^{(1)}, \ldots, y_{\gamma}^{(n)}\right)= & \sum_{s=0}^{n} y_{\gamma}^{(s)} \frac{\partial F}{\partial y^{(s)}}\left(y_{\gamma}, y_{\gamma}^{(1)}, \ldots, y_{\gamma}^{(n)}\right) \\
& -F\left[y_{\gamma}, y_{\gamma}^{(1)}, \ldots, y_{\gamma}^{(n)}\right]+\psi(x),
\end{aligned}
$$

$$
\mathscr{G}_{1}\left[y, y^{(1)}, \ldots, y^{(n)}\right]=G\left[y, y^{(1)}, \ldots, y^{(n)}\right] .
$$

Here $\mathscr{G}_{1}$ is a nonlinear function that is decomposed using the spectral homotopy analysis method $[15,16]$. We define the following zeroth-order deformation equations:

$$
(1-q) \mathscr{L}_{1}\left[\bar{Y}(x ; q)-\bar{y}_{0}(x)\right]=q \hbar\left\{\mathcal{N}_{1}[\bar{Y}(x ; q)]-\Phi\left(\bar{y}_{\gamma}\right)\right\},
$$

where $q \in[0,1]$ is an embedding parameter, $\bar{Y}(x ; q)$ are unknown functions, $\hbar$ is the convergence controlling parameter, and the "bar" has been introduced for convenience to denote the associated function and its $n$ derivatives. For example,

$$
\bar{y} \equiv\left(y, y^{(1)}, y^{(2)}, \ldots, y^{(n)}\right)
$$


The nonlinear operator $\mathcal{N}_{1}$ is defined by

$$
\mathscr{N}_{1}[\bar{Y}(x ; q)]=\mathscr{L}_{1}\left[\bar{Y}_{r}(t ; q)\right]+\mathscr{G}_{1}[\bar{Y}(x ; q)] .
$$

By differentiating the zeroth-order equations (11) $m$ times with respect to $q$, setting $q=0$, and finally dividing the resulting equations by $m$ ! (see, e.g., [17, 30-32]), we obtain the following $m$ th order deformation equations:

$$
\begin{array}{r}
\mathscr{L}_{1}\left[\bar{y}_{m}(x)-\left(\chi_{m}+\hbar\right) \bar{y}_{m-1}(x)\right] \\
=\hbar R_{m-1}\left[\bar{y}_{0}, \bar{y}_{1}, \ldots, \bar{y}_{m-1}\right],
\end{array}
$$

where

$$
\begin{gathered}
R_{m-1}\left[\bar{y}_{0}, \bar{y}_{1}, \ldots, \bar{y}_{m-1}\right] \\
=\left.\frac{1}{(m-1) !} \frac{\partial^{m-1}\left\{\mathscr{G}_{1}[\bar{Y}(x ; q)]-\Phi\left(\bar{y}_{\gamma}\right)\right\}}{\partial q^{m-1}}\right|_{q=0}, \\
\chi_{m}= \begin{cases}0, & m \leqslant 1, \\
1, & m>1 .\end{cases}
\end{gathered}
$$

After obtaining solutions for (12), the approximate solution for $y(x)$ is determined as the series solution

$$
y(x)=\sum_{m=0}^{\infty} y_{m}(x) .
$$

The SHAM solution is said to be of order $M$ if the previous series is truncated at $m=M$, that is, if

$$
y(x)=\sum_{m=0}^{M} y_{m}(x) .
$$

The initial approximation $\bar{y}_{0}$ required for solving the sequence of linear higher order deformation equations (12) is chosen as the solution that results from solving the linear part of (5) subject to the given boundary conditions (2). That is, we solve

$$
\mathscr{L}_{1}\left[y_{0}, y_{0}^{(1)}, \ldots, y_{0}^{(n)}\right]=\Phi\left(y_{\gamma}, y_{\gamma}^{(1)}, \ldots, y_{\gamma}^{(n)}\right) .
$$

We note that with $\mathscr{L}_{1}$ as defined in (6), (16) cannot be solved exactly by means of analytical techniques. Numerical methods such as finite differences, finite element method, and spectral method can be used to solve equations of the form (16). Thus, if only the initial approximation is used to approximate the solution $y(x)$ of the governing nonlinear differential equation (1), that is, if $y(x) \approx y_{0}(x)$, the $(r+1)$ th approximation of (1) is a solution of

$$
\mathscr{L}_{1}\left[y_{r+1}, y_{r+1}^{(1)}, \ldots, y_{r+1}^{(n)}\right]=\Phi\left(y_{\gamma}, y_{\gamma}^{(1)}, \ldots, y_{\gamma}^{(n)}\right)
$$

which, on using the definitions (6) and (7), can be written as

$$
\begin{aligned}
& L\left[y_{r+1}, y_{r+1}^{(1)}, \ldots, y_{r+1}^{(n)}\right] \\
& \quad+\sum_{s=0}^{n}\left(y_{r+1}^{(s)}-y_{r}^{(s)}\right) \frac{\partial F}{\partial y^{(s)}}\left(y_{r}, y_{r}^{(1)}, \ldots, y_{r}^{(n)}\right) \\
& \quad+F\left[y_{r}, y_{r}^{(1)}, \ldots, y_{r}^{(n)}\right]=0 .
\end{aligned}
$$

We note that the iterative scheme (18) is, in fact, the quasilinearization method of Bellman and Kalaba [1]. For $M=1$, we have

$$
y(x) \approx y_{0}(x)+y_{1}(x),
$$

where $y_{1}$ is obtained as a solution of

$$
\begin{aligned}
& \mathscr{L}_{1}\left[y_{1}, y_{1}^{(1)}, \ldots, y_{1}^{(n)}\right] \\
& \quad=\hbar \mathscr{L}_{1}\left[y_{0}, y_{0}^{(1)}, \ldots, y_{0}^{(n)}\right]+\hbar R_{0}\left[y_{0}, y_{0}^{(1)}, \ldots, y_{0}^{(n)}\right] .
\end{aligned}
$$

This produces the iteration scheme

$$
\begin{aligned}
& \mathscr{L}_{1}\left[y_{r+1}, \ldots, y_{r+1}^{(n)}\right] \\
& =\Phi\left[y_{r}, y_{r}^{(1)}, \ldots, y_{r}^{(n)}\right]+\hbar \mathscr{L}_{1}\left[y_{0, r+1}, \ldots, y_{0, r+1}^{(n)}\right] \\
& \quad+\hbar R_{0}\left[y_{0, r+1}, \ldots, y_{0, r+1}^{(n)}\right],
\end{aligned}
$$

where

$$
\begin{aligned}
\mathscr{L}_{1}\left[y_{r+1}, y_{r+1}^{(1)}, \ldots, y_{r+1}^{(n)}\right]= & L\left[y_{r+1}, y_{r+1}^{(1)}, \ldots, y_{r+1}^{(n)}\right] \\
& +\sum_{s=0}^{n} y_{r+1}^{(s)} \frac{\partial F}{\partial y^{(s)}}\left(y_{r}, y_{r}^{(1)}, \ldots, y_{r}^{(n)}\right)
\end{aligned}
$$

and $y_{0, r+1}$ is the solution of

$$
\begin{aligned}
& L\left[y_{0, r+1}, y_{0, r+1}^{(1)}, \ldots, y_{0, r+1}^{(n)}\right]+\sum_{s=0}^{n} y_{0, r+1}^{(s)} \frac{\partial F}{\partial y^{(s)}}\left(y_{r}, y_{r}^{(1)}, \ldots, y_{r}^{(n)}\right) \\
& =\Phi\left(y_{r}, y_{r}^{(1)}, \ldots, y_{r}^{(n)}\right] .
\end{aligned}
$$

For $M=2$, we have

$$
y(x) \approx y_{0}(x)+y_{1}(x)+y_{2}(x),
$$

where $y_{2}$ is obtained as a solution of

$$
\begin{aligned}
\mathscr{L}_{1}\left[y_{2}, y_{2}^{(1)}, \ldots, y_{2}^{(n)}\right] & \\
= & (1+\hbar) \mathscr{L}_{1}\left[y_{1}, y_{1}^{(1)}, \ldots, y_{1}^{(n)}\right] \\
& +\hbar R_{1}\left[y_{0}, y_{0}^{(1)}, \ldots, y_{0}^{(n)} ; y_{1}, y_{1}^{(1)}, \ldots, y_{1}^{(n)}\right] .
\end{aligned}
$$

This produces the iteration scheme

$$
\begin{aligned}
\mathscr{L}\left[\bar{y}_{r+1}\right]= & \Phi\left[\bar{y}_{r}\right]+\hbar \mathscr{L}_{1}\left[\bar{y}_{0, r+1}\right]+(1+\hbar) \mathscr{L}_{1}\left[\bar{y}_{1, r+1}\right] \\
& +\hbar\left\{R_{0}\left[\bar{y}_{0, r+1}\right]+R_{1}\left[\bar{y}_{0, r+1}, \bar{y}_{1, r+1}\right]\right\},
\end{aligned}
$$

where $y_{1, r+1}$ is obtained as the solution of

$$
\mathscr{L}_{1}\left[\bar{y}_{1, r+1}\right]=\hbar \mathscr{L}_{1}\left[\bar{y}_{0, r+1}\right]+\hbar R_{0}\left[\bar{y}_{0, r+1}\right] .
$$


In general, for any $M>1$, we have

$$
y(x)=y_{0}(x)+y_{1}(x)+\cdots+y_{M}(x),
$$

where $y_{M}(x)$ is obtained as a solution of

$$
\mathscr{L}_{1}\left[\bar{y}_{M}\right]=(1+\hbar) \mathscr{L}_{1}\left[\bar{y}_{M-1}\right]+\hbar R_{M-1}\left[\bar{y}_{0}, \bar{y}_{1}, \ldots, \bar{y}_{M-1}\right] \text {. }
$$

Thus, a general scheme when the SHAM is truncated at order $M$ (where $M \geq 1$ ), hereinafter referred to as scheme- $M$, can be obtained as

$$
\begin{aligned}
& \mathscr{L}_{1}\left[y_{r+1}, y_{r+1}^{(1)}, \ldots, y_{r+1}^{(n)}\right] \\
& =\Phi\left[y_{r}, y_{r}^{(1)}, \ldots, y_{r}^{(n)}\right] \\
& \quad+\sum_{p=0}^{M-1}\left(\chi_{p+1}+\hbar\right) \mathscr{L}_{1}\left[y_{p, r+1}, y_{p, r+1}^{(1)}, \ldots, y_{p, r+1}^{(n)}\right] \\
& +\hbar \sum_{p=0}^{M-1} R_{p}\left[\bar{y}_{0, r+1}, \bar{y}_{1, r+1}, \ldots, \bar{y}_{p, r+1}\right],
\end{aligned}
$$

where each $y_{p, r+1}$ is obtained as the solution of

$$
\begin{aligned}
& \mathscr{L}_{1}\left[\bar{y}_{p, r+1}\right] \\
& =\left\{\begin{array}{cc}
\left(\chi_{p}+\hbar\right) \mathscr{L}_{1}\left[\bar{y}_{p-1, r+1}\right] & \\
+\hbar R_{p-1}\left[\bar{y}_{0, r+1}, \bar{y}_{1, r+1}, \ldots, \bar{y}_{p-1, r+1}\right] & \text { when } p \geq 1, \\
\Phi\left[y_{r}, y_{r}^{(1)}, \ldots, y_{r}^{(n)}\right] & \text { when } p=0 .
\end{array}\right.
\end{aligned}
$$

\section{Solution of the Falkner-Skan Equation}

In this section we demonstrate how the numerical schemes derived in the previous section may be used to solve the Falkner-Skan equation:

$$
\begin{aligned}
& f^{\prime \prime \prime}(\eta)+\beta f(\eta) f^{\prime \prime}(\eta)+\beta_{1}\left(1-f^{\prime}(\eta)^{2}\right) \\
& =0, \quad \eta \in[0, \infty),
\end{aligned}
$$

subject to the boundary conditions

$$
f(0)=f^{\prime}(0)=0, \quad \lim _{\eta \rightarrow \infty} f(\eta)=1 .
$$

It is convenient to first define

$$
F\left(y, y^{\prime}, y^{\prime \prime}\right)=\beta y y^{\prime \prime}-\beta_{1}\left(y^{\prime}\right)^{2}, \quad \Psi=-\beta_{1},
$$

so that

$$
\begin{gathered}
\Phi\left(y, y^{\prime}, y^{\prime \prime}\right)=\beta y y^{\prime \prime}-\beta_{1}\left(y^{\prime}\right)^{2}-\beta_{1} \\
\mathscr{L}_{1}\left(y, y^{\prime}, y^{\prime \prime}, y^{\prime \prime \prime}\right)=y^{\prime \prime \prime}+a_{0, \gamma} y^{\prime \prime}+a_{1, \gamma} y^{\prime}+a_{2, \gamma} y \\
R_{m-1}\left[\bar{y}_{0}, \bar{y}_{1}, \ldots, \bar{y}_{m-1}\right]= \\
\beta \sum_{j=0}^{m-1} y_{j} y_{m-1-j}^{\prime \prime}-\beta_{1} \sum_{j=0}^{m-1} y_{j}^{\prime} y_{m-1-j}^{\prime} \\
+\beta_{1}\left(1-\chi_{m}\right)+2 \beta_{1} y_{\gamma}^{\prime} y_{m-1}^{\prime} \\
-\beta\left(y_{\gamma} y_{m-1}^{\prime \prime}+y_{\gamma}^{\prime \prime} y_{m-1}\right)
\end{gathered}
$$

where

$$
a_{0, \gamma}=\beta y_{\gamma}, \quad a_{1, \gamma}=-2 \beta_{1} y_{\gamma}^{\prime}, \quad a_{2, \gamma}=\beta y_{\gamma}^{\prime \prime} .
$$

Using (35)-(37) the first three iterative schemes corresponding to $M=0,1,2$ may now be defined as follows.

3.1. Scheme-0. In this scheme we set

$$
\mathscr{L}_{1}\left(y_{r+1}, y_{r+1}^{\prime}, y_{r+1}^{\prime \prime}, y_{r+1}^{\prime \prime \prime}\right)=\Phi\left(y_{r}, y_{r}^{\prime}, y_{r}^{\prime \prime}\right)
$$

subject to

$$
y_{r+1}(0)=y_{r+1}^{\prime}(0)=0, \quad y_{r+1}^{\prime}(\infty)=1 .
$$

It is worth noting that Scheme- 0 is, in fact, equivalent to the original QLM algorithm; see Mandelzweig and Tabakin [5] and Mandelzweig [6].

3.2. Scheme-1. For this scheme we set

$$
\begin{aligned}
& \mathscr{L}_{1}\left(y_{r+1}, y_{r+1}^{\prime}, y_{r+1}^{\prime \prime}, y_{r+1}^{\prime \prime \prime}\right) \\
& =\Phi\left(y_{r}, y_{r}^{\prime}, y_{r}^{\prime \prime}\right)+\hbar \mathscr{L}_{1}\left(y_{0, r+1}, y_{0, r+1}^{\prime}, y_{0, r+1}^{\prime \prime}, y_{0, r+1}^{\prime \prime \prime}\right) \\
& \quad+\hbar R_{0}\left(y_{0, r+1}, y_{0, r+1}^{\prime}, y_{0, r+1}^{\prime \prime}\right),
\end{aligned}
$$

subject to

$$
y_{r+1}(0)=y_{r+1}^{\prime}(0)=0, \quad y_{r+1}^{\prime}(\infty)=1,
$$

where

$$
\begin{aligned}
R_{0} & \left(y_{0, r+1}, y_{0, r+1}^{\prime}, y_{0, r+1}^{\prime \prime}\right) \\
= & \beta y_{0, r+1} y_{0, r+1}^{\prime \prime}-\beta_{1} y_{0, r+1}^{\prime 2}+\beta_{1}+2 \beta_{1} y_{r}^{\prime} y_{0, r+1}^{\prime} \\
& -\beta\left(y_{r} y_{0, r+1}^{\prime \prime}+y_{r}^{\prime \prime} y_{0, r+1}\right),
\end{aligned}
$$

and $y_{0, r+1}$ is the solution of

$$
\mathscr{L}_{1}\left(y_{0, r+1}, y_{0, r+1}^{\prime}, y_{0, r+1}^{\prime \prime}, y_{0, r+1}^{\prime \prime \prime}\right)=\Phi\left(y_{r}, y_{r}^{\prime}, y_{r}^{\prime \prime}\right)
$$

subject to

$$
y_{0, r+1}(0)=y_{0, r+1}^{\prime}(0)=0, \quad y_{0, r+1}^{\prime}(\infty)=1 .
$$

3.3. Scheme-2. The complexity of the defining equations increases with the order of the scheme. For Scheme-2 we have

$$
\begin{aligned}
& \mathscr{L}_{1}\left(y_{r+1}, y_{r+1}^{\prime}, y_{r+1}^{\prime \prime}, y_{r+1}^{\prime \prime \prime}\right) \\
&= \Phi\left(y_{r}, y_{r}^{\prime}, y_{r}^{\prime \prime}\right)+\hbar \mathscr{L}_{1}\left(y_{0, r+1}, y_{0, r+1}^{\prime}, y_{0, r+1}^{\prime \prime}, y_{0, r+1}^{\prime \prime \prime}\right) \\
&+(1+\hbar) \mathscr{L}_{1}\left(y_{1, r+1}, y_{1, r+1}^{\prime}, y_{1, r+1}^{\prime \prime}, y_{1, r+1}^{\prime \prime \prime}\right) \\
&+\hbar R_{0}\left(y_{0, r+1}, y_{0, r+1}^{\prime}, y_{0, r+1}^{\prime \prime}\right) \\
&+\hbar R_{1}\left(y_{0, r+1}, y_{0, r+1}^{\prime}, y_{0, r+1}^{\prime \prime} ; y_{1, r+1}, y_{1, r+1}^{\prime}, y_{1, r+1}^{\prime \prime}\right),
\end{aligned}
$$


subject to

$$
y_{r+1}(0)=y_{r+1}^{\prime}(0)=0, \quad y_{r+1}^{\prime}(\infty)=1,
$$

where

$$
\begin{aligned}
R_{1}\left(y_{0, r+1}, y_{0, r+1}^{\prime}, y_{0, r+1}^{\prime \prime} ; y_{1, r+1}, y_{1, r+1}^{\prime}, y_{1, r+1}^{\prime \prime}\right) \\
=\beta\left(y_{0, r+1} y_{1, r+1}^{\prime \prime}+y_{1, r+1} y_{0, r+1}^{\prime \prime}\right)-2 \beta_{1} y_{0, r+1}^{\prime} y_{1, r+1} \\
\quad+2 \beta_{1} y_{r}^{\prime} y_{1, r+1}^{\prime}-\beta\left(y_{r} y_{1, r+1}^{\prime \prime}+y_{r}^{\prime \prime} y_{1, r+1}\right)
\end{aligned}
$$

and $y_{1, r+1}$ is the solution of

$$
\begin{gathered}
\mathscr{L}_{1}\left(y_{1, r+1}, y_{1, r+1}^{\prime}, y_{1, r+1}^{\prime \prime}, y_{1, r+1}^{\prime \prime \prime}\right) \\
=\hbar \mathscr{L}_{1}\left(y_{0, r+1}, y_{0, r+1}^{\prime}, y_{0, r+1}^{\prime \prime}, y_{0, r+1}^{\prime \prime \prime}\right) \\
+\hbar R_{0}\left(y_{0, r+1}, y_{0, r+1}^{\prime}, y_{0, r+1}^{\prime \prime}\right),
\end{gathered}
$$

with

$$
y_{1, r+1}(0)=y_{1, r+1}^{\prime}(0)=0, \quad y_{1, r+1}^{\prime}(\infty)=0 .
$$

Equations (39), (41), and (46) describing the three solution schemes can be solved numerically using standard methods such as finite difference, finite elements, and spline collocation methods. In this study we use the Chebyshev spectral collocation method to solve the iteration schemes, (see [33-36]). To allow for numerical implementation of the pseudospectral method, the physical region $[0, \infty)$ is truncated to $[0, L]$ where $L$ is chosen to be sufficiently large. The truncated region is further transformed to the space $[-1,1]$ using the transformation

$$
\xi=\frac{2}{L_{\infty}} \eta-1 .
$$

As with any other numerical approximation method, some sort of discretization is introduced in the interval $[-1,1]$. We choose the Gauss-Lobatto collocation points to define the nodes in $[-1,1]$ as

$$
\xi_{j}=\cos \left(\frac{\pi j}{N}\right), \quad j=0,1, \ldots, N,
$$

where $(N+1)$ is the number of collocation points. The essence of the Chebyshev spectral collocation method is the idea of introducing a differentiation matrix $D$. The differentiation matrix maps a vector of the function values $\mathbf{Y}=\left[y\left(\xi_{0}\right), \ldots, y\left(\xi_{N}\right)\right]^{T}$ at the collocation points to a vector $\mathbf{Y}^{\prime}$ defined as

$$
\mathbf{Y}^{\prime}=\sum_{k=0}^{N} D_{j k} f\left(\xi_{k}\right)=D \mathbf{Y}
$$

In general, a derivative of order $p$ for the function $y(\eta)$ can be expressed as

$$
y^{(p)}(\eta)=\mathbf{D}^{p} \mathbf{Y}
$$

where $\mathbf{D}=2 D / L_{\infty}$. The matrix $D$ is of size $(N+1) \times(N+1)$ and its entries are defined as

$$
\begin{aligned}
D_{j k} & =\frac{c_{j}}{c_{k}} \frac{(-1)^{j+k}}{\tau_{j}-\tau_{k}}, \quad j \neq k ; j, k=0,1, \ldots, N, \\
D_{k k} & =-\frac{\tau_{k}}{2\left(1-\tau_{k}^{2}\right)}, \quad k=1,2, \ldots, N-1, \\
D_{00} & =\frac{2 N^{2}+1}{6}=-D_{N N},
\end{aligned}
$$

with

$$
c_{k}= \begin{cases}2 & k=0, N \\ 1 & -1 \leq k \leq N-1 .\end{cases}
$$

Thus, applying the spectral method to the iteration Scheme0 (39) and the corresponding boundary conditions gives the following matrix system:

$$
\mathbf{A}_{r} \mathbf{Y}_{r+1}=\Phi_{r}
$$

with boundary conditions

$$
\begin{gathered}
y_{r+1}\left(\xi_{N}\right)=0, \quad \sum_{k=0}^{N} \mathbf{D}_{N k} y_{r+1}\left(\xi_{k}\right)=0, \\
\sum_{k=0}^{N} \mathbf{D}_{0 k} y_{r+1}\left(\xi_{k}\right)=1,
\end{gathered}
$$

where

$$
\mathbf{A}_{r}=\mathbf{D}^{3}+\mathbf{a}_{0, r} \mathbf{D}^{2}+\mathbf{a}_{1, r} \mathbf{D}+\mathbf{a}_{2, r},
$$

where $\Phi_{r}$ corresponds to the function $\Phi\left(y, y, y^{\prime \prime}\right)$ when evaluated at the collocation points and $\mathbf{a}_{i, r}(i=0,1,2)$ is a diagonal matrix corresponding to the vector of $a_{i, r}$.

The boundary conditions (58) are imposed on the first, $N$ th, and $(N+1)$ th rows of $A_{r}$ and $\Phi_{r}$ to obtain a system of the form

$$
\left(\begin{array}{ccccc}
\mathbf{D}_{0,0} & \mathbf{D}_{0,1} & \cdots & \mathbf{D}_{0, N-1} & \mathbf{D}_{0, N} \\
& & \mathbf{A}_{\mathbf{r}} & & \\
& & & & \\
\mathbf{D}_{N, 0} & \mathbf{D}_{N, 1} & \cdots & \mathbf{D}_{N, N-1} & \mathbf{D}_{N, N} \\
0 & 0 & \cdots & 0 & 1
\end{array}\right)\left(\begin{array}{c}
y_{r+1}\left(\xi_{0}\right) \\
y_{r+1}\left(\xi_{1}\right) \\
\vdots \\
y_{r+1}\left(\xi_{N-2}\right) \\
y_{r+1}\left(\xi_{N-1}\right) \\
y_{r+1}\left(\xi_{N}\right)
\end{array}\right)
$$

$$
=\left(\begin{array}{c}
1 \\
\Phi_{r}\left(\xi_{1}\right) \\
\vdots \\
\Phi_{r}\left(\xi_{N-2}\right) \\
0 \\
0
\end{array}\right) .
$$


TABLE 1: Comparison between the computed values of the skin friction $f^{\prime \prime}(0)$ for the Blasius flow $\left(\beta=1 / 2, \beta_{1}=0\right)$.

\begin{tabular}{|c|c|c|}
\hline iter. & $f^{\prime \prime}(0)$ & Error \\
\hline & \multicolumn{2}{|c|}{ SHAM } \\
\hline 1 & 0.361245275076317576714175486031 & 0.02918793886112127777699542402 \\
\hline 10 & 0.332060294018222201221920675183 & 0.00000295780302590228474061317 \\
\hline 20 & 0.332057337274670714006530763206 & 0.00000000105947441506935070120 \\
\hline 30 & 0.332057336215760109582560249181 & 0.00000000000056381064538018717 \\
\hline 40 & 0.332057336215196653344046405966 & 0.00000000000000035440686634396 \\
\hline \multirow[t]{2}{*}{60} & 0.332057336215196298937262729762 & 0.00000000000000000000008266775 \\
\hline & \multicolumn{2}{|c|}{ Scheme-0 } \\
\hline 1 & 0.36124527510805664031836423508 & 0.02918793889286034138118417307 \\
\hline 2 & 0.33293906079206190667160822082 & 0.00088172457686560773442815881 \\
\hline 3 & 0.33205878995514977263006366166 & 0.00000145373995347369288359965 \\
\hline 4 & 0.33205733621994973222724960736 & 0.00000000000475343329006954535 \\
\hline 5 & 0.33205733621519629893723540415 & 0.00000000000000000000005534214 \\
\hline \multirow[t]{2}{*}{6} & 0.33205733621519629893718006201 & 0.00000000000000000000000000000 \\
\hline & \multicolumn{2}{|c|}{ Scheme-1 } \\
\hline 1 & 0.33849743020925601396026175681 & 0.00644009399405971502308169480 \\
\hline 2 & 0.33205889444389263880627992358 & 0.00000155822869633986909986157 \\
\hline 3 & 0.33205733621519633877777093517 & 0.00000000000000003984059087316 \\
\hline \multirow[t]{2}{*}{4} & 0.33205733621519629893718006201 & 0.00000000000000000000000000000 \\
\hline & \multicolumn{2}{|c|}{ Scheme-2 } \\
\hline 1 & 0.33398877527020321822942828158 & 0.00193143905500691929224821957 \\
\hline 2 & 0.33205733679309573625968418056 & 0.00000000057789943732250411855 \\
\hline 3 & 0.33205733621519629893718006201 & 0.00000000000000000000000000000 \\
\hline \multicolumn{3}{|c|}{ [29]: 0.33205733621519629893718006201 (104 iterations) } \\
\hline
\end{tabular}

TABLE 2: Comparison between the computed values of the skin friction $f^{\prime \prime}(0)$ for the Pohlhausen flow $\left(\beta=0, \beta_{1}=1\right)$.

\begin{tabular}{|c|c|c|}
\hline iter. & $f^{\prime \prime}(0)$ & Error \\
\hline & \multicolumn{2}{|c|}{ SHAM } \\
\hline 1 & 1.15819390472196206795661617787 & 0.00349336634271053893831861687 \\
\hline 5 & 1.15470068226816961126259064868 & 0.00000014388891808224429308768 \\
\hline 10 & 1.15470053837716000145607336675 & 0.00000000000209152756222419425 \\
\hline 20 & 1.15470053837925152901716901667 & 0.00000000000000000000112854434 \\
\hline 25 & 1.15470053837925152901829759222 & 0.00000000000000000000000003121 \\
\hline \multirow[t]{2}{*}{30} & 1.15470053837925152901829756100 & 0.00000000000000000000000000000 \\
\hline & \multicolumn{2}{|c|}{ Scheme-0 } \\
\hline 1 & 1.1581939047219620679566161779 & 0.0034933663427105389383186169 \\
\hline 2 & 1.1547034510528929300844093465 & 0.0000029126736414010661117855 \\
\hline 3 & 1.1547005383817023293095010719 & 0.0000000000024508002912035109 \\
\hline 4 & 1.1547005383792515290182994735 & 0.0000000000000000000000019125 \\
\hline \multirow[t]{2}{*}{5} & 1.1547005383792515290182975610 & 0.0000000000000000000000000000 \\
\hline & \multicolumn{2}{|c|}{ Scheme-1 } \\
\hline 1 & 1.1544901934962778016810840055 & 0.0002103448829737273372135555 \\
\hline 2 & 1.1547005383778620432865956388 & 0.0000000000013894857317019222 \\
\hline 3 & 1.1547005383792515290182975610 & 0.0000000000000000000000000000 \\
\hline
\end{tabular}

EXACT: 1.1547005383792515290182975610

[29]: 1.1547005383792515290182975610 (104 iterations) 
TABle 3: Comparison between the computed values of the skin friction $f \prime \prime(0)$ for the Homann flow $\left(\beta=2, \beta_{1}=1\right)$.

\begin{tabular}{|c|c|c|}
\hline iter. & $f^{\prime \prime}(0)$ & Error \\
\hline & \multicolumn{2}{|c|}{ SHAM } \\
\hline 1 & 1.335633919867798255673072885920 & 0.023696225987993120191426715220 \\
\hline 10 & 1.311933330446726656235176983180 & 0.000004363433078479246469187519 \\
\hline 20 & 1.311937690198936556741381203220 & 0.000000003680868578740264967481 \\
\hline 30 & 1.311937693875056506843830283790 & 0.000000000004748628637815886910 \\
\hline 40 & 1.311937693879797836016540925840 & 0.000000000000007299465105244860 \\
\hline 50 & 1.311937693879805123127203300860 & 0.000000000000000012354442869842 \\
\hline 60 & 1.311937693879805135459418974120 & 0.000000000000000000022227196583 \\
\hline \multirow[t]{2}{*}{70} & 1.311937693879805135481601938190 & 0.000000000000000000000044232511 \\
\hline & \multicolumn{2}{|c|}{ Scheme-0 } \\
\hline 1 & 1.3356339198662404626942038769 & 0.0236962259864353272125577062 \\
\hline 2 & 1.3121878643609977795027970207 & 0.0002501704811926440211508500 \\
\hline 3 & 1.3119377351892323008775816635 & 0.0000000413094271653959354928 \\
\hline 4 & 1.3119376938798066169255039256 & 0.0000000000000014814438577549 \\
\hline \multirow[t]{2}{*}{5} & 1.3119376938798051354816461707 & 0.0000000000000000000000000000 \\
\hline & \multicolumn{2}{|c|}{ Scheme-1 } \\
\hline 1 & 1.3064680181439000004910779728 & 0.0054696757359051349905681979 \\
\hline 2 & 1.3119375731135522096139489286 & 0.0000001207662529258676972421 \\
\hline 3 & 1.3119376938798051354785690801 & 0.0000000000000000000030770906 \\
\hline \multirow[t]{2}{*}{4} & 1.3119376938798051354816461707 & 0.0000000000000000000000000000 \\
\hline & \multicolumn{2}{|c|}{ Scheme-2 } \\
\hline 1 & 1.3136564701352183697646278650 & 0.0017187762554132342829816943 \\
\hline 2 & 1.3119376938938730064725895040 & 0.0000000000140678709909433333 \\
\hline 3 & 1.3119376938798051354816461707 & 0.0000000000000000000000000000 \\
\hline \multicolumn{3}{|c|}{ [29]: 1.3119376938798051354816461707 (104 iterations) } \\
\hline
\end{tabular}

Starting from a suitable initial guess $y_{0}(\eta)$, the iteration scheme (60) can be used to iteratively give approximate solutions of the governing equation (32) for Scheme-0. The application of the pseudospectral method for Scheme-1 and Scheme-2 can be done in a similar manner. The initial approximation used in all the algorithms is

$$
y_{0}(\eta)=\eta+e^{-\eta}+1 \text {. }
$$

The number of collocation points used in all the results presented here is $N=200$ with $L_{\infty}=20$.

\section{Results and Discussion}

In this section we present solutions of the Falkner-Skan equation (32) using the QLM-SHAM hybrid iteration schemes. Numerical simulations were conducted for the following special classes of the F-S equations:

(i) Blasius flow: $\beta=1 / 2, \beta_{1}=0$,

(ii) Pohlhausen flow: $\beta=0, \beta_{1}=1$,

(iii) Homann flow: $\beta=2, \beta_{1}=1$.

To assess the accuracy and performance of our schemes, the numerical results were compared to the recently reported results of Ganapol [29]. To date, these results are the most accurate results for the Blasius and Falkner-Skan class of equations. Ganapol [29] reported highly accurate results between 10 and 30 decimal places using a robust algorithm based on Maclaurin series with convergence acceleration and analytical continuation techniques.

The comparison between the present findings and the results in the literature is made for the skin friction which is proportional to $f^{\prime \prime}(0)$. Table 1 shows a comparison between the computed skin friction values of the Blasius equation using the three QLM-SHAM iteration schemes. The results are compared with the results reported in Ganapol [29] which are accurate to 29 decimal places. We observe that all the iteration schemes rapidly converge to the results of [29] to all 29 decimal places. Full convergence is achieved after 6 iterations when using Scheme-0, 4 iterations when using Scheme-1, and after 3 iterations when using Scheme-2. It is worth noting that the results of [29] were achieved after 104 decimal places. Prior to Ganapol [29], the most accurate Blasius skin friction results had been published to 17 decimal places by Boyd [28] as $f^{\prime \prime}(0)=0.33205733621519630$. This result was obtained after 5 iterations using Scheme- 0 and 3 iterations for both Schemes-1 and -2 . The value reported after 52 iterations in [29] is $f^{\prime \prime}(0)=0.3320573362151965$. It is clear that the proposed iteration schemes converge significantly faster than the method of [29]. That the results 
of Boyd [28] and Ganapol [29] were obtained only after a few iterations validates both the higher order convergence and the accuracy of the present solution methods.

Table 2 shows the computed values of $f^{\prime \prime}(0)$ using Schemes- 0 and -1 for the Pohlhausen flow $\left(\beta=0, \beta_{1}=1\right)$. For this particular flow, the exact value of $f^{\prime \prime}(0)$ is known to be $2 / \sqrt{3}$. The iteration Scheme- 0 matches the exact result after only 5 iterations and Scheme-1 converges after only 3 iterations. The method used in Ganapol [29] converged to the exact result after 104 iterations. This again demonstrates the superior convergence of the present method.

Table 3 gives the numerical simulations of the skin friction results for the Homann flow. We observe that the 29-digit results reported in [29] are achieved in 5 iterations, 4 iterations, and 3 iterations for Scheme- 0 , -1 , and -2 , respectively. This result indicates that adding an additional level in the QLM-SHAM scheme would further significantly increase the convergence of the iteration scheme. We further note from Tables 1-3 that all three schemes converge significantly much faster than the spectral homotopy analysis method on its own.

\section{Conclusion}

In this study we presented three hybrid QLM-SHAM iteration schemes for the solution of Falkner-Skan type boundary layer equations. We have shown through numerical experimentation that the proposed numerical schemes significantly enhance the convergence rate of the quasilinearization method. By comparison with the most accurate solutions of the Falkner-Skan equations currently available in the literature, we have shown that the schemes are highly accurate and efficient in terms of the number of iterations required to determine the solution to the required level of accuracy. The schemes presented provide robust tools for the efficient solution of nonlinear equations by offering superior accuracy to many existing methods. In addition, the approach used in deriving these schemes provides a suitable framework for extension to higher level schemes by adding more terms of the SHAM component of the method.

\section{References}

[1] R. E. Bellman and R. E. Kalaba, Quasilinearization and Nonlinear Boundary-Value Problems, Elsevier, New York, NY, USA, 1965.

[2] K. Maleknejad and E. Najafi, "Numerical solution of nonlinear Volterra integral equations using the idea of quasilinearization," Communications in Nonlinear Science and Numerical Simulation, vol. 16, no. 1, pp. 93-100, 2011.

[3] R. Krivec and V. B. Mandelzweig, "Numerical investigation of quasilinearization method in quantum mechanics," Computer Physics Communications, vol. 138, pp. 69-79, 2001.

[4] V. B. Mandelzweig, "Quasilinearization method and its verification on exactly solvable models in quantum mechanics," Journal of Mathematical Physics, vol. 40, no. 12, pp. 6266-6291, 1999.

[5] V. B. Mandelzweig and F. Tabakin, "Quasilinearization approach to nonlinear problems in physics with application to nonlinear ODEs," Computer Physics Communications, vol. 141, no. 2, pp. 268-281, 2001.
[6] V. B. Mandelzweig, "Quasilinearization method: nonperturbative approach to physical problems," Physics of Atomic Nuclei, vol. 68, no. 7, pp. 1227-1258, 2005.

[7] V. Lakshmikantham, S. Leela, and S. Sivasundaram, "Extensions of the method of quasilinearization," Journal of Optimization Theory and Applications, vol. 87, no. 2, pp. 379-401, 1995.

[8] V. Lakshmikantham, "Further improvement of generalized quasilinearization method," Nonlinear Analysis. Theory, Methods \& Applications, vol. 27, no. 2, pp. 223-227, 1996.

[9] K. Parand, M. Ghasemi, S. Rezazadeh, A. Peiravi, A. Ghorbanpour, and A. Tavakoli Golpaygani, "Quasilinearization approach for solving Volterra's population model," Applied and Computational Mathematics, vol. 9, no. 1, pp. 95-103, 2010.

[10] J. Jiang and A. S. Vatsala, "The quasilinearization method in the system of reaction diffusion equations," Applied Mathematics and Computation, vol. 97, no. 2-3, pp. 223-235, 1998.

[11] B. Ahmad, "A quasilinearization method for a class of integrodifferential equations with mixed nonlinearities," Nonlinear Analysis. Real World Applications, vol. 7, no. 5, pp. 997-1004, 2006.

[12] S. G. Pandit, "Quadratically converging iterative schemes for nonlinear Volterra integral equations and an application," Journal of Applied Mathematics and Stochastic Analysis, vol. 10, no. 2, pp. 169-178, 1997.

[13] J. I. Ramos, "Piecewise-quasilinearization techniques for singularly perturbed Volterra integro-differential equations," Applied Mathematics and Computation, vol. 188, no. 2, pp. 1221-1233, 2007.

[14] S. Tuffuor and J. W. Labadie, "A nonlinear time variant rainfallrunoff model for augmenting monthly data," Water Resources Research, vol. 10, pp. 1161-1166, 1974.

[15] S. S. Motsa, P. Sibanda, and S. Shateyi, "A new spectralhomotopy analysis method for solving a nonlinear second order BVP," Communications in Nonlinear Science and Numerical Simulation, vol. 15, no. 9, pp. 2293-2302, 2010.

[16] S. S. Motsa, P. Sibanda, F. G. Awad, and S. Shateyi, "A new spectral-homotopy analysis method for the MHD JefferyHamel problem," Computers \& Fluids, vol. 39, no. 7, pp. 12191225, 2010.

[17] S. J. Liao, Beyond Perturbation: Introduction to Homotopy Analysis Method, Chapman \& Hall/CRC Press, 2003.

[18] S. Li and S.-J. Liao, "An analytic approach to solve multiple solutions of a strongly nonlinear problem," Applied Mathematics and Computation, vol. 169, no. 2, pp. 854-865, 2005.

[19] P. Sibanda, S. Motsa, and Z. Makukula, "A spectral-homotopy analysis method for heat transfer flow of a third grade fluid between parallel plates," International Journal of Numerical Methods for Heat \& Fluid Flow, vol. 22, no. 1, pp. 4-23, 2012.

[20] S. S. Motsa and P. Sibanda, "On the solution of MHD flow over a nonlinear stretching sheet by an efficient semi-analytical technique," International Journal for Numerical Methods in Fluids, vol. 68, no. 12, pp. 1524-1537, 2012.

[21] S. Abbasbandy, "Improving Newton-Raphson method for nonlinear equations by modified Adomian decomposition method," Applied Mathematics and Computation, vol. 145, no. 2-3, pp. 887-893, 2003.

[22] C. Chun, "Iterative methods improving Newton's method by the decomposition method," Computers \& Mathematics with Applications, vol. 50, no. 10-12, pp. 1559-1568, 2005.

[23] G. Adomian, "A review of the decomposition method and some recent results for nonlinear equations," Mathematical and Computer Modelling, vol. 13, no. 7, pp. 17-43, 1990. 
[24] G. Adomian and R. Rach, "Noise terms in decomposition solution series," Computers \& Mathematics with Applications, vol. 24, no. 11, pp. 61-64, 1992.

[25] G. Adomian, Solving Frontier Problems of Physics: The Decomposition Method, Kluwer Academic Publishers Group, Boston, Mass, USA, 1994.

[26] C. Chun, "Construction of Newton-like iteration methods for solving nonlinear equations," Numerische Mathematik, vol. 104, no. 3, pp. 297-315, 2006.

[27] M. A. Noor, "Iterative methods for nonlinear equations using homotopy perturbation technique," Applied Mathematics \& Information Sciences, vol. 4, no. 2, pp. 227-235, 2010.

[28] J. P. Boyd, “The Blasius function: computations before computers, the value of tricks, undergraduate projects, and open research problems," SIAM Review, vol. 50, no. 4, pp. 791-804, 2008.

[29] B. D. Ganapol, "Highly accurate solutions of the Blasiusand Falkner-Skan boundary layer equations via convergence acceleration," http://arxiv.org/abs/1006.3888.

[30] A. S. Bataineh, M. S. M. Noorani, and I. Hashim, "Series solution of the multispecies Lotka-Volterra equations by means of the homotopy analysis method," Differential Equations and Nonlinear Mechanics, vol. 2008, Article ID 816787, 14 pages, 2008.

[31] A. S. Bataineh, M. S. M. Noorani, and I. Hashim, "Homotopy analysis method for singular IVPs of Emden-Fowler type," Communications in Nonlinear Science and Numerical Simulation, vol. 14, no. 4, pp. 1121-1131, 2009.

[32] Y. Tan and S. Abbasbandy, "Homotopy analysis method for quadratic Riccati differential equation," Communications in Nonlinear Science and Numerical Simulation, vol. 13, no. 3, pp. 539-546, 2008.

[33] C. Canuto, M. Y. Hussaini, A. Quarteroni, and T. A. Zang, Spectral Methods in Fluid Dynamics, Springer, Berlin, Germany, 1988.

[34] W. S. Don and A. Solomonoff, "Accuracy and speed in computing the Chebyshev collocation derivative," SIAM Journal on Scientific Computing, vol. 16, no. 6, pp. 1253-1268, 1995.

[35] L. N. Trefethen, Spectral Methods in MATLAB, SIAM, 2000.

[36] J. A. C. Weideman and S. C. Reddy, "A MATLAB differentiation matrix suite," ACM Transactions on Mathematical Software, vol. 26, no. 4, pp. 465-519, 2000. 


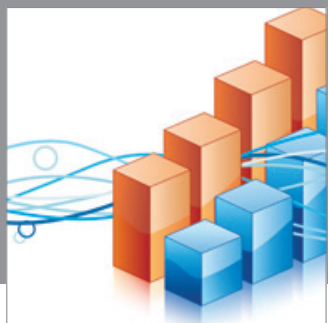

Advances in

Operations Research

mansans

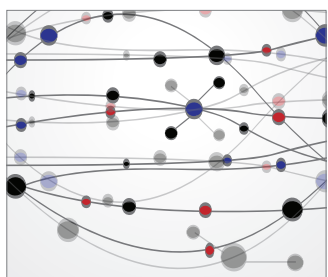

The Scientific World Journal
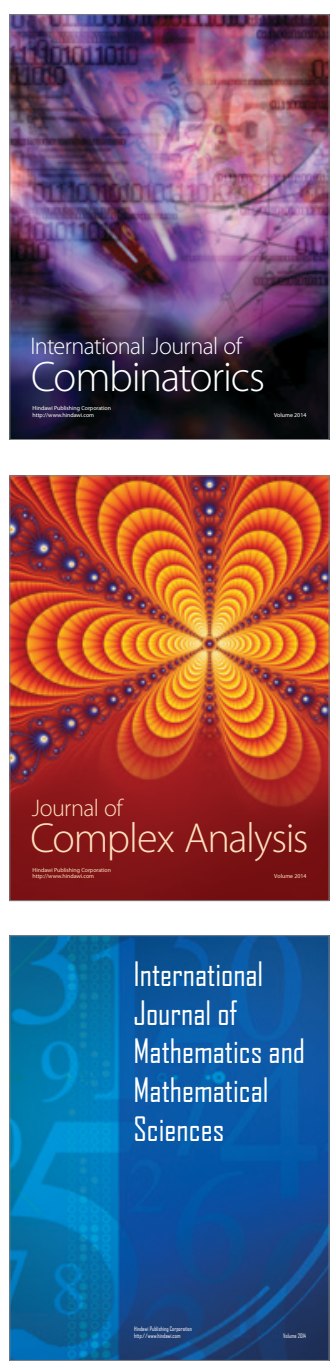
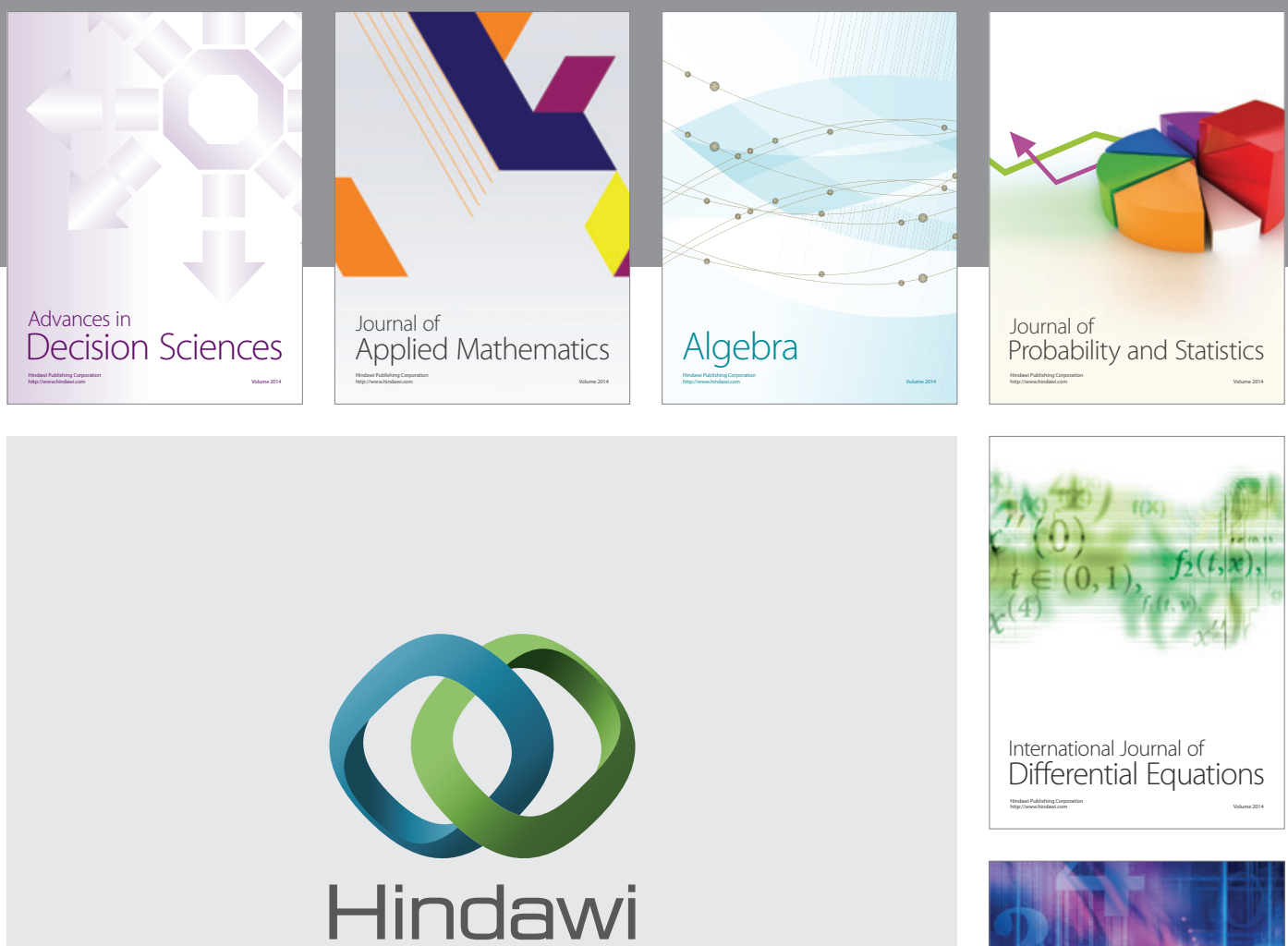

Submit your manuscripts at http://www.hindawi.com
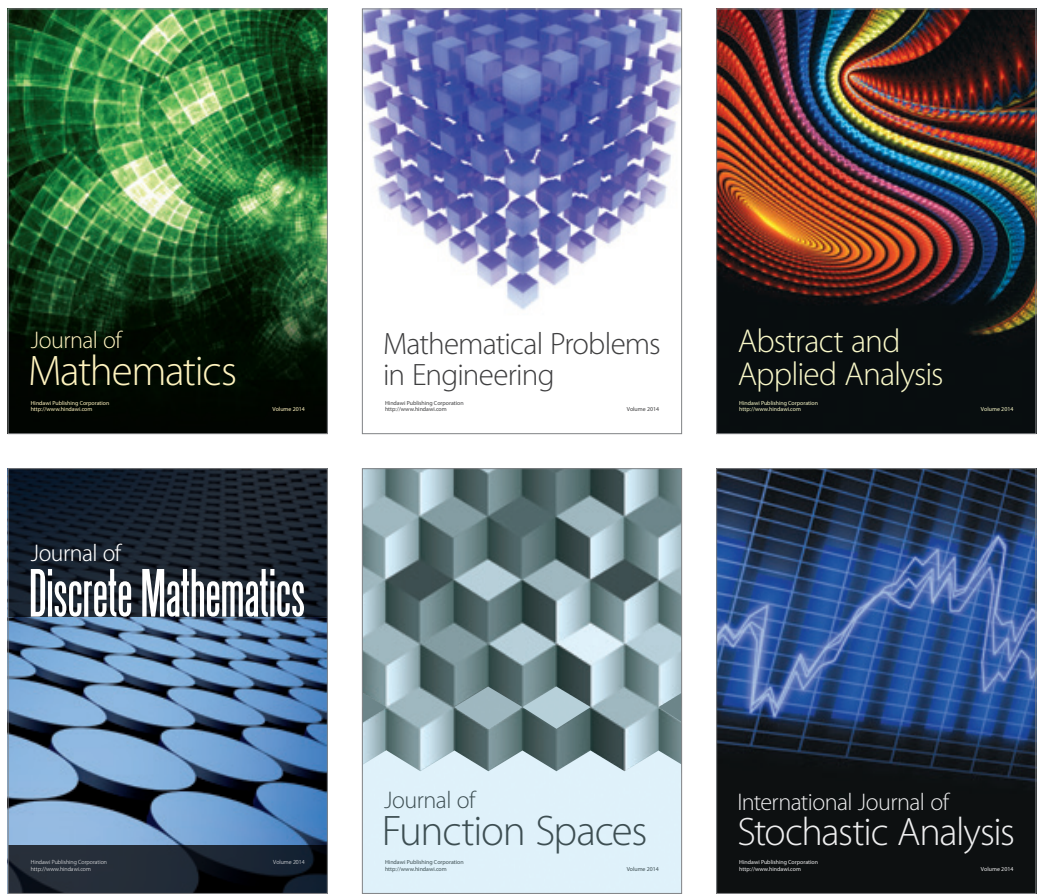

Journal of

Function Spaces

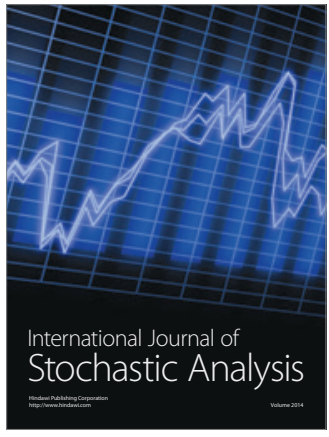

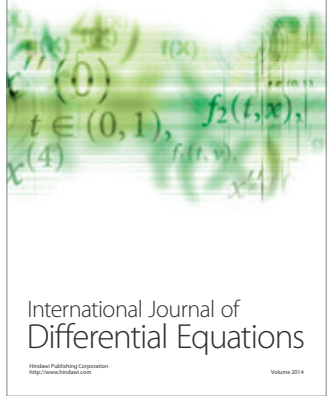
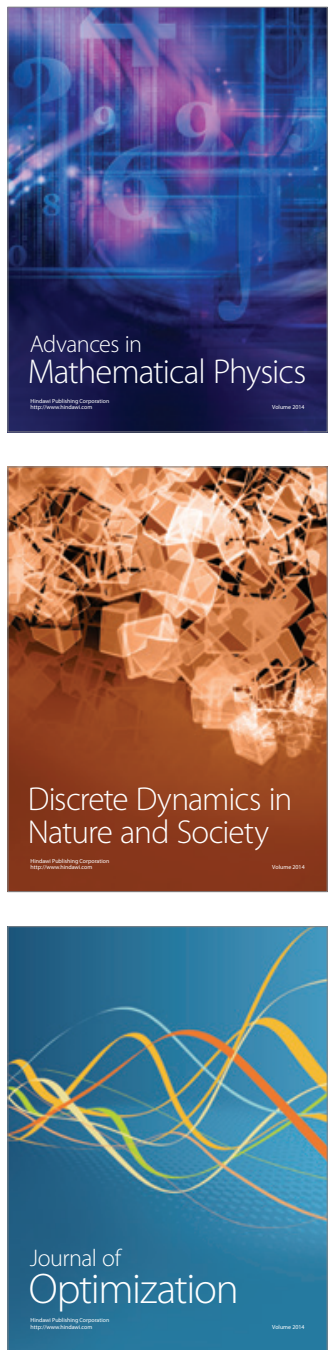